\title{
Redes Sociais Significativas de Mulheres com Transtornos Alimentares
}

\section{Significant Social Nets of Women with Eating Disorders}

\author{
Carolina Leonidas* \& Manoel Antônio dos Santos \\ Universidade de São Paulo, Ribeirão Preto, São Paulo, Brasil
}

\begin{abstract}
Resumo
O presente artigo teve como objetivo investigar como se configuram as redes sociais significativas de mulheres com transtornos alimentares (TAs), o apoio social percebido e suas possíveis implicações para a evolução do quadro. Foram investigadas seis mulheres cadastradas em um serviço de assistência em TA da região sudeste do Brasil. Os instrumentos utilizados foram Mapa de Rede e entrevista semiestruturada. Os relatos foram submetidos à análise de conteúdo temática. Evidenciou-se que as relações interpessoais consideradas significativas eram muito escassas e apresentavam baixa qualidade em termos de grau do envolvimento e apoio mútuo, comprometendo o potencial de apoio e, consequentemente, o bem-estar das participantes. A família caracterizou-se como a principal fonte de apoio social. Concluindo, há necessidade de incluir as redes de apoio na assistência, a fim de fortalecê-las para prevenir agravos.

Palavras-chave: Transtornos alimentares, redes sociais, apoio social.
\end{abstract}

\begin{abstract}
This article aims to investigate how the significant social networks of women with eating disorders (ED) are configured, as well as the perceived social support and its possible implications on the evolution of the disorder. Six women in follow-up at an assistance service in ED in southeastern Brazil were investigated. The instruments used were the Network's Map and semi-structured interview. The reports were submitted to thematic content analysis. It was evident that interpersonal relationships considered significant were scarce and with poor quality in terms of involvement degree and mutual support, weakening the potential support and, consequently, the participants'welfare and mental health. The family was characterized as the main source of social support. It is necessary to include support networks in health care and strengthening them in order to prevent diseases.

Keywords: Eating disorders, social networks, social support.
\end{abstract}

Os transtornos alimentares (TAs) são quadros psicopatológicos caracterizados por graves perturbações no comportamento alimentar, que afetam, em sua maioria, adolescentes e jovens do sexo feminino (T. F. Andrade \& Santos, 2009; Scorsolini-Comin, Souza, \& Santos, 2010). Esses quadros podem resultar em prejuízos biológicos, psicológicos e sociais, que acarretam aumento da morbidade e mortalidade. Anorexia nervosa (AN) e bulimia nervosa (BN) são os tipos mais prevalentes de TAs, segundo o
DSM-IV-TR ${ }^{T M}$ - Manual Diagnóstico e Estatístico de Transtornos Mentais, $4^{a}$ edição (Associação Americana de Psiquiatria, 2003).

O DSM-IV-TR ${ }^{\mathrm{TM}}$ caracteriza a AN em termos de uma recusa do indivíduo a manter o peso corporal na faixa normal mínima, de acordo com sua idade e altura. $\mathrm{O}$ quadro envolve um temor intenso de ganhar peso e uma perturbação significativa na percepção da forma ou tamanho do corpo, o que resulta em caquexia, ou seja, fraqueza geral e má disposição corporal decorrente da desnutrição (Claudino \& Borges, 2002). Já a BN consiste em repetições de episódios de compulsão alimentar (binge eating), nos quais o indivíduo ingere grandes quantidades de alimento em curto período de tempo, vivenciando uma sensação de perda de controle (Rosa \& Santos, 2011). São praticados exercícios físicos excessivos, dietas rigorosas, indução de vômitos e uso abusivo de laxantes, diuréticos e anorexígenos, devido à preocupação obsessiva com o peso e a forma corporal, bem como a busca de alívio após a ingestão compulsiva de alimento (Kaye, 2008; Paccola, 2006). 
No que concerne aos aspectos de personalidade, as mulheres com AN apresentam baixa autoestima, sentimentos de inferioridade e inadequação pessoal, insegurança, perfeccionismo e obsessividade, fatores que acarretam acentuada inibição e retraimento social, que geralmente aparecem em comorbidade com transtornos de ansiedade e do humor (Borges, Sicchieri, Ribeiro, Marchini, \& Santos, 2006; Cassin \& Von Ranson, 2005; Castro-Fornieles et al., 2007; Nilsson, Sundbom, \& Häglöf, 2008). As mulheres com BN, em contrapartida, apresentam comportamentos marcadamente impulsivos, autoestima flutuante, pensamentos e emoções desadaptativas, busca exacerbada de estímulos e experiências novas, além de traços histriônicos, entre outros, sendo comum o diagnóstico de transtorno de personalidade do tipo borderline como comorbidade psiquiátrica (Cassin \& Von Ranson, 2005).

TA é uma categoria psicopatológica ainda cercada por amplo desconhecimento por parte da população em relação às suas características, evolução e tratamento, o que acaba gerando incompreensão por parte dos familiares, amigos, professores e colegas de trabalho (Souza \& Santos, 2007, 2009, 2010). Por ser uma condição crônica estigmatizante, esse desconhecimento incrementa o isolamento social, intensificando os sentimentos de solidão e desamparo da pessoa acometida. Por essa razão, ao planejar o cuidado a esses pacientes, é fundamental que os profissionais conheçam sua rede social e o apoio percebido (Vieira \& Nóbrega, 2004; Resende, Bones, Souza, \& Guimarães, 2006).

\section{Marco Teórico: Redes e Apoio Social}

Teoria das Redes Sociais (Sluzki, 1997) trata do "conjunto de seres com quem interagimos de maneira regular, com quem conversamos, com quem trocamos sinais que nos corporizam, que nos tornam reais" (p. 15). Segundo este autor, a identidade é construída e reconstruída constantemente ao longo do ciclo vital, a partir da interação do indivíduo com os outros (familiares, vizinhos, amigos, inimigos, conhecidos, companheiros de grupos, entre outros). Assim, considera-se que esses "outros" são parte intrínseca da identidade de cada pessoa.

As redes sociais têm, como característica primária, o fato de serem abertas e dinâmicas, ou seja, são marcadas pelo inacabamento, estão em permanente construção e têm como princípio básico a conectividade (Custódio, 2010; Moré, 2005; Orlandi, 2011). Dessa forma, pode-se dizer que as redes sociais possibilitam as trocas efetivas entre o indivíduo e os integrantes de entidades coletivas. É por meio de tais intercâmbios que os recursos desses grupos podem ser utilizados de forma a facilitar a resolução de conflitos e a satisfação de necessidades do indivíduo. Além disso, levando-se em conta o caráter provisório e o processo de constante reconstrução das redes, Orlandi (2011) aponta para a impossibilidade de representá-las em sua totalidade. Resta apenas ao observador da rede visualizar a forma como ela está configurada naquele determinado momento, "considerando a sua volatilidade e o contexto de observação" (p. 38).
Sluzki (1997) utiliza o termo "rede social pessoal" ou "rede social significativa" para caracterizar algumas relações particulares que fazem parte do microssistema do indivíduo, ou seja, que estão inseridas em seu ambiente imediato e que o influenciam de forma direta. $\mathrm{O}$ autor concebe a rede social pessoal como "a soma de todas as relações que um indivíduo percebe como significativas ou define como diferenciadas da massa anônima da sociedade" (p. 42). Assim, Sluzki (1997) estabelece uma diferenciação entre os vários ambientes que integram as inúmeras relações que o indivíduo estabelece (Custódio, 2010). O referido autor acrescenta, ainda, que as redes sociais pessoais contribuem para que o indivíduo se reconheça como tal e possa desenvolver sua autoimagem. Ao desenvolver um senso de identidade, bem-estar, competência e autoria, o indivíduo também pode se responsabilizar de forma mais ativa pelos cuidados à sua saúde e pela adaptação às situações de crise (Sluzki, 1997). Moré (2005), por sua vez, utiliza a terminologia de "rede pessoal significativa" para denominar essas mesmas relações, descritas por Sluzki (1997) como aquelas que são diferenciadas da massa de pessoas que circundam o indivíduo. Tendo em vista a necessidade de uniformização dos termos utilizados no presente estudo, optou-se por fazer uso da terminologia adotada por Sluzki (1997), ou seja, "rede social significativa".

As redes sociais significativas podem ser analisadas a partir de algumas características, definidas por Sluzki (1997). Dentre elas, encontram-se:

1. Estrutura, ou seja, as propriedades da rede em seu conjunto, que englobam: tamanho, densidade, composição, dispersão e homogeneidade/heterogeneidade dos membros;

2. Funcionalidade, que diz respeito ao tipo predominante de intercâmbio interpessoal característico de vínculos específicos, que podem ser definidos como: companhia social, apoio emocional, guia cognitivo e de conselhos, regulação social, ajuda material e de serviços e acesso a novos contatos; e

3. Atributos dos vínculos, que são as propriedades específicas de cada relação: função predominante do vínculo, multidimensionalidade, reciprocidade, intensidade ou compromisso da relação, frequência de contatos e história da relação.

De forma complementar à Teoria das Redes Sociais, o presente estudo se apóia no referencial de Apoio Social de Bullock (2004). Essa autora sugere que as redes sociais são geralmente compreendidas em termos estruturais, descritas como as interações que o indivíduo estabelece com as instituições sociais que o cercam, tais como família, vizinhança e organizações comunitárias e religiosas. Já a noção de apoio social, por sua vez, enfatiza as trocas interpessoais estabelecidas entre alguns membros da rede. Nesse sentido, o apoio social tem sido considerado como parte importante da promoção de saúde, uma vez que fornece assistência às necessidades físicas e emocionais 
do indivíduo, assim como ajuda a amenizar o efeito que eventos estressores exercem sobre a qualidade de vida (Bullock, 2004).

$\mathrm{O}$ conceito de rede social encontra-se estreitamente articulado ao construto de apoio social. Segundo Resende et al. (2006), apoio social pode ser definido como um sistema de relações formais e informais pelas quais os indivíduos recebem ajuda emocional, material ou de informação para enfrentar situações geradoras de tensão emocional. Deve ser compreendido como uma experiência pessoal e subjetiva, que leva a um maior senso de satisfação com a vida, favorecendo a organização da identidade por meio do reconhecimento do indivíduo pelo olhar do outro, possibilitando que ele sinta que "esta aí para alguém" (G. R. B. Andrade \& Vaitsman, 2002).

Segundo G. R. B. Andrade e Vaitsman (2002), a condição de enfermidade implica no enfrentamento, por parte do indivíduo acometido, de limitações, impedimentos e situações que afetam as relações com as pessoas que compõem a rede social. Silva et al. (2003) mencionam a existência de uma relação entre apoio social e uma variedade de indicadores e medidas dependentes, tais como: saúde, adaptação psicológica, percepção de bem-estar, redução do mal-estar, longevidade e mortalidade, satisfação com a vida, entre outros. As influências positivas das redes sociais na saúde podem ser compreendidas pela constatação de que elas favorecem o monitoramento da saúde por meio da convivência com as pessoas (G. R. B. Andrade \& Vaitsman, 2002). Além disso, a disposição para enfrentar os desafios da vida, lutar pelos direitos e pôr em prática projetos viáveis dentro das condições pessoais e do meio ambiente em que se vive, também parece ser respaldada por uma rede de apoio social (Marcos \& Cantero, 2009; Resende et al., 2006).

$\mathrm{O}$ entendimento da rede social significativa do sujeito acometido por TAs é fundamental, especialmente quando se pretende compreender as teias de conflito, o potencial familiar de apoio em situação de crise, o núcleo relacional a ser ativado para uma melhor inserção social do indivíduo, entre outros (Vieira \& Nóbrega, 2004).

Atualmente, muitas pesquisas a respeito das redes familiares de apoio têm documentado o impacto do apoio social sobre os comportamentos saudáveis, tanto na prevenção quanto no tratamento dos agravos à saúde (Bullock, 2004). Ao pensar essa interface do apoio e das redes sociais com a promoção de saúde, a autora sugere que esses construtos estão associados com a redução das taxas de mortalidade, com a melhora de doenças graves e com o aumento da utilização de práticas preventivas de cuidado à saúde. Ao considerar a relação entre apoio social e saúde, a referida autora define o conceito de apoio como o fornecimento ou recebimento de assistência, e sugere que a percepção que o indivíduo tem desse apoio pode ser tanto positiva quanto negativa.

A temática das redes sociais é de relevância atual e contribui com a possibilidade efetiva de uma leitura ampliada dos processos diagnósticos psicopatológicos, levando em conta as implicações da trama relacional em que as pessoas estão inseridas. Tal reconhecimento tem repercussão direta nas possibilidades de intervenção junto aos indivíduos acometidos por condições crônicas de saúde.

Acredita-se que a constituição da identidade pessoal do sujeito se dá no espaço intersubjetivo das relações familiares, sociais e pessoais, e no contato com as diversas redes as quais ele é exposto no decorrer do ciclo vital. Partindo-se da hipótese de que as relações que as mulheres com TAs estabelecem ao longo de sua vida são bastante conturbadas e fragilizadas, marcadas por conflitos e por dificuldades no estabelecimento de vínculos consistentes (Abreu \& Cangelli, 2004; Leonidas \& Santos, 2012; Oliveira \& Santos, 2006; Peres \& Santos, 2006), pode-se postular que suas redes sociais são marcadas por pouco envolvimento dos membros e baixa qualidade dos vínculos, o que comprometeria o potencial de apoio e o próprio desenvolvimento do senso de identidade. A baixa efetividade das redes e o comprometimento do apoio social disponibilizado a essas mulheres podem agravar os sintomas de TAs, intensificando a cronicidade do quadro psicopatológico. Nesse sentido, considera-se que a inclusão das redes sociais significativas na assistência a pacientes acometidos por condições crônicas, tais como os TAs, é um passo essencial para ampliar as possibilidades de oferta de cuidado terapêutico, favorecendo a efetividade da ajuda prestada pelos cuidadores profissionais.

Considerando esses pressupostos, o presente estudo teve como objetivo investigar o modo como se configuram as redes sociais de mulheres com diagnóstico de TAs, assim como o apoio social percebido por elas e suas possíveis implicações para a evolução do quadro psicopatológico.

\section{M étodo}

Trata-se de um estudo do tipo exploratório e descritivo, com enfoque qualitativo.

\section{Participantes}

Foram incluídas no estudo seis mulheres jovens e adultas. Os critérios de inclusão foram: (a) faixa etária entre 20 e 30 anos, adotado para oferecer maior homogeneidade à amostra; (b) ter diagnóstico de TAs; (c) estar cadastrada no Grupo de Assistência em Transtornos Alimentares - GRATA, do Hospital das Clínicas da Faculdade de Medicina de Ribeirão Preto da Universidade de São Paulo (HC-FMRP-USP); (d) estar frequentando o serviço no período da coleta de dados. As variáveis sociodemográficas das participantes estão sistematizados na Tabela 1 e os dados antropométricos na Tabela 2. Como mostra a Tabela 1, a idade média das participantes foi 26,17 anos. No que concerne à categoria diagnóstica (Tabela 2), três das participantes apresentavam quadro de $\mathrm{BN}$ e três apresentavam AN. 
Psicologia: Reflexão e Crítica, 26(3), 561-571.

Tabela 1

Caracterização das Participantes segundo Idade, Escolaridade, Profissão/Ocupação, Estado Marital e Número de Filhos no Momento da Entrevista

\begin{tabular}{lccccc}
\hline Participantes* & $\begin{array}{c}\text { Idade } \\
\text { (anos) }\end{array}$ & $\begin{array}{c}\text { Estado } \\
\text { Marital }\end{array}$ & Filhos & Escolaridade & Profissão/Ocupação \\
\hline Paula & 30 & Separada & - & Ensino superior completo & Auxiliar administrativa \\
Grazieli & 20 & Solteira & - & Ensino médio completo & Vendedora em banca de revistas \\
Sissi & 27 & Solteira & - & Ensino superior incompleto & Estudante universitária \\
Denise & 29 & Separada & Dois & Ensino médio completo & Auxiliar administrativa \\
Juliana & 27 & Solteira & - & Ensino médio completo & $\begin{array}{c}\text { Atendente de telemarketing } \\
\text { (afastada) }\end{array}$ \\
Tatiana & 24 & Solteira & - & Ensino superior incompleto & Estudante universitária \\
\hline
\end{tabular}

Nota. *Para preservar o anonimato das participantes, todos os nomes utilizados neste estudo são fictícios e foram escolhidos pelas próprias entrevistadas.

Tabela 2

Caracterização das Participantes segundo o Diagnóstico, Peso, Altura, Índice de Massa Corporal (IMC) na Admissão e no Momento da Avaliação, e Tempo de Tratamento no Serviço

\begin{tabular}{lcccccc}
\hline Participantes & Diagnóstico & Peso (kg) & Altura (m) & $\begin{array}{c}\text { IMC de admissão } \\
\left(\mathrm{kg} / \mathrm{m}^{2}\right)\end{array}$ & $\begin{array}{c}\text { IMC atual }(\mathrm{kg} / \\
\left.\mathrm{m}^{2}\right)\end{array}$ & $\begin{array}{c}\text { Tempo de tratamento } \\
\text { no serviço (meses) }\end{array}$ \\
\hline Paula & AN & 49,1 & 1,64 & 17,8 & 18,3 & 22 \\
Grazieli & BN & 59,3 & 1,65 & 23,5 & 21,9 & 10 \\
Sissi & BN & 63,4 & 1,75 & 21,2 & 20,6 & 12 \\
Denise & BN & 60,7 & 1,68 & 20,1 & 21,5 & 51 \\
Juliana & AN & 44,1 & 1,72 & 14,7 & 14,9 & 6 \\
Tatiana & AN & 56,4 & 1,60 & 21,9 & 21,9 & 1 \\
\hline
\end{tabular}

No período do estudo 15 pacientes frequentavam o serviço, 12 com diagnóstico de AN e três com BN. A interrupção da coleta obedeceu ao critério de saturação dos dados, comumente adotado em pesquisas qualitativas (Guest, Bunce, \& Johnson, 2006). Esse critério preconiza que o número ideal de sujeitos é atingido na medida em que as respostas começam a apresentar repetições quando se adicionam novos participantes no estudo, não acrescendo informações novas que possam ser úteis e relevantes para o alcance dos objetivos.

\section{Instrumentos}

Para a coleta de dados foram utilizados os seguintes instrumentos: roteiro de entrevista semiestruturada e Mapa de Rede. Também foram consultados os prontuários individuais. Vale ressaltar que o Mapa de Rede foi utilizado em função da potencialidade gráfica que oferece, uma vez que possibilita melhor integração das informações oriundas dos relatos das participantes durante as entrevistas (Santos, 2009). Dessa forma, considera-se que o Mapa de Rede e os prontuários hospitalares foram recursos utilizados de forma complementar à entrevista semiestruturada.

Roteiro de Entrevista Semiestruturada. A técnica de entrevista semiestruturada foi utilizada por permitir o aprofundamento no tema de pesquisa. Foi composto um roteiro de perguntas elaboradas de forma a atender aos objetivos da investigação, adotando uma formulação flexível, de modo que a sucessão das questões e os detalhes deveriam ficar por conta do discurso das participantes e da dinâmica da entrevista. A entrevistadora introduzia o tema e a entrevistada tinha ampla abertura para discorrer sobre ele, podendo assim explorar livremente a questão. As perguntas foram respondidas dentro de uma conversação informal, na qual a interferência da entrevistadora foi a mínima possível (Minayo, 1994). O roteiro da entrevista semiestruturada abrangeu: 
Leonidas, C. \& Santos, M. A. (2013). Redes Sociais Significativas de Mulheres com Transtornos Alimentares.

1. Histórico do TA;

2. Informações relativas ao tratamento;

3. Situação emocional da participante;

4. Rede social e pessoal da participante; e

5. Rede familiar. Os dois últimos itens (4 e 5) foram constituídos de questões específicas para montagem do Mapa de Rede.

Mapa de Rede. Trata-se de um instrumento elaborado por Sluzki (1997), que consiste em representar a rede so- cial do indivíduo a partir de um mapa dividido em quatro quadrantes, relacionados à família, às amizades, às relações de trabalho ou escola, às relações comunitárias e às relações com instituições (como serviço de saúde e jurídico) ou de credo religioso. O mapa é dividido em círculos de proximidade, caracterizados por "relações íntimas", "sociais" e "conhecidos". A forma como o Mapa de Rede é configurado pode ser visualizada na Figura 1, conforme proposta de Santos (2009).

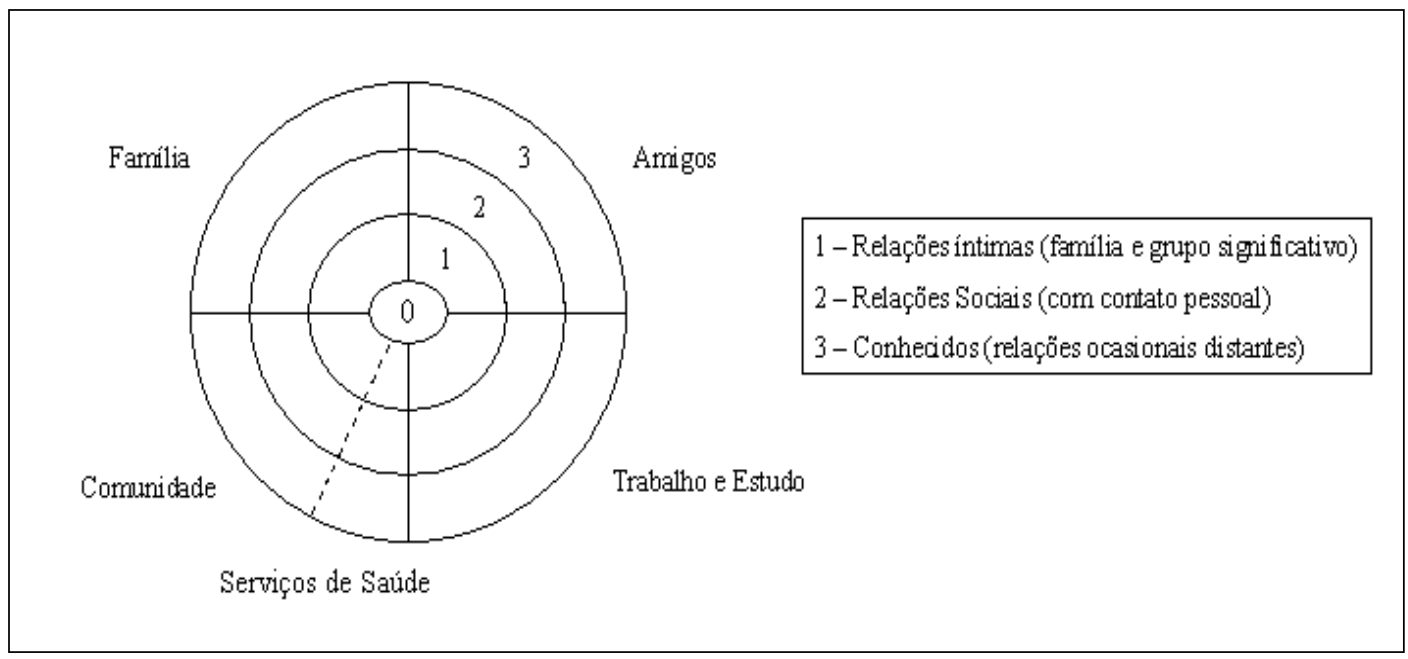

Figura 1. Mapa de Rede proposto por Sluzki (1997).

Para fins do presente estudo, a representação gráfica da participante foi colocada no centro do círculo e, no lugar dos Serviços de Saúde, conforme modelo sugerido por Sluzki (1997), foi inserido o serviço especializado no tratamento dos TAs (GRATA).

Como já explicitado anteriormente, as questões utilizadas na montagem do Mapa de Rede foram incorporadas ao roteiro de entrevista, nos itens 4 e 5 . Assim, a construção dos mapas foi realizada conjuntamente pela pesquisadora e pelas participantes. A pesquisadora anotou todos os nomes mencionados pelas participantes. Foram identificados o tipo de relação que a participante mantinha com aquelas pessoas, de modo a facilitar a escolha da localização em que elas deveriam ser inseridas nos mapas. Dessa forma, foi possível visualizar, graficamente, a forma como as redes eram estruturadas, assim como as funções desempenhadas pelos membros e os atributos dos vínculos (Sluzki, 1997). Para fins de apresentação dos resultados, todos os nomes próprios foram substituídos por pseudônimos.

Prontuários Hospitalares. Foram feitas consultas para obtenção dos dados sociodemográficos (idade, estado civil, escolaridade e ocupação) e antropométricos (diagnóstico, peso, altura, índice de massa corporal e tempo de tratamento) das participantes.

\section{Considerações Éticas}

O estudo foi aprovado pelo Comitê de Ética em Pesquisa do Hospital das Clínicas da Faculdade de Medicina de
Ribeirão Preto da Universidade de São Paulo (HC-FMRP-USP), processo HCRP nº 2155/2010.

\section{Procedimento}

Coleta de Dados. Após receberem os devidos esclarecimentos a respeito da pesquisa, as participantes assinaram o Termo de Consentimento Livre e Esclarecido. A aplicação dos instrumentos foi realizada individualmente, no decorrer de um único encontro com duração de $40 \mathrm{a}$ 60 minutos, em sala reservada da instituição hospitalar, no período de maio a novembro de 2010. A entrevista foi audiogravada e, posteriormente, transcrita na íntegra e de forma literal. O Mapa de Rede foi construído juntamente com a aplicação da entrevista, que continha as questões referentes à confecção do instrumento. Foi construído pela própria pesquisadora a partir dos dados fornecidos pela participante.

Análise de Dados. O material transcrito e o Mapa de Rede constituíram o corpus da pesquisa. Os relatos foram submetidos à análise de conteúdo na modalidade temática (Bogdan \& Biklen, 1994). Os dados foram agrupados em categorias relacionadas às configurações das redes sociais das participantes e à percepção que elas tinham do apoio recebido por meio dessas redes. Os resultados foram analisados com base na Teoria das Redes Sociais de Sluzki (1997) e do Apoio Social de Bullock (2004). 


\section{Resultados e Discussão}

A partir da análise das entrevistas e dos Mapas de Rede elaborados para cada participante, evidenciou-se, de modo geral, que as relações interpessoais consideradas significativas eram muito escassas e apresentavam baixa qualidade em termos de grau do envolvimento e apoio mútuo. Essas relações englobavam, basicamente, alguns membros da família imediata (sobretudo a mãe, o pai e os irmãos), poucos amigos íntimos e alguns profissionais do serviço de saúde, principalmente psicólogos e nutricionistas.

A configuração dos Mapas de Rede indicou, no mínimo, cinco e, no máximo, sete pessoas no círculo de "Relações Íntimas". Poucas pessoas foram inseridas no círculo de "Relações Sociais" e um número menor ainda no último círculo, que corresponde aos "Conhecidos". Nesse último círculo, as participantes incluíram um ex-namorado, pacientes do GRATA, profissionais do GRATA, "pessoas da faculdade", "pessoas da igreja" e "pessoas do trabalho". Essas pessoas foram grafadas entre aspas porque não foram nomeadas pelas participantes, impossibilitando a individualização e quantificação do número de relações.

As "Relações Íntimas" eram constituídas, principalmente, por amigos próximos (da infância e/ou adolescência, da faculdade, do trabalho e/ou da internet, e vizinhas), pela família, principalmente filhos, mãe, pai e irmãos, pelos psicólogos e nutricionistas do GRATA, por algumas pessoas da igreja. Por fim, as "Relações Sociais" abarcavam tios, sobrinhos, cunhados, irmãos e avós, o pai, amigas da faculdade, orientadora de iniciação científica, uma paciente do GRATA, psicóloga, nutricionista e psiquiatra do GRATA. A Figura 2 possibilita a visualização da distribuição dos membros das redes das participantes nos quadrantes dos Mapas de Rede. Vale ressaltar que se tratam de números aproximados, uma vez que, como já mencionado anteriormente, algumas participantes referiram, de forma genérica, "pessoas", "primos", "tios" e/ ou "amigas", sem discriminar um número específico de indivíduos, o que impossibilitou a quantificação exata de membros das redes sociais.

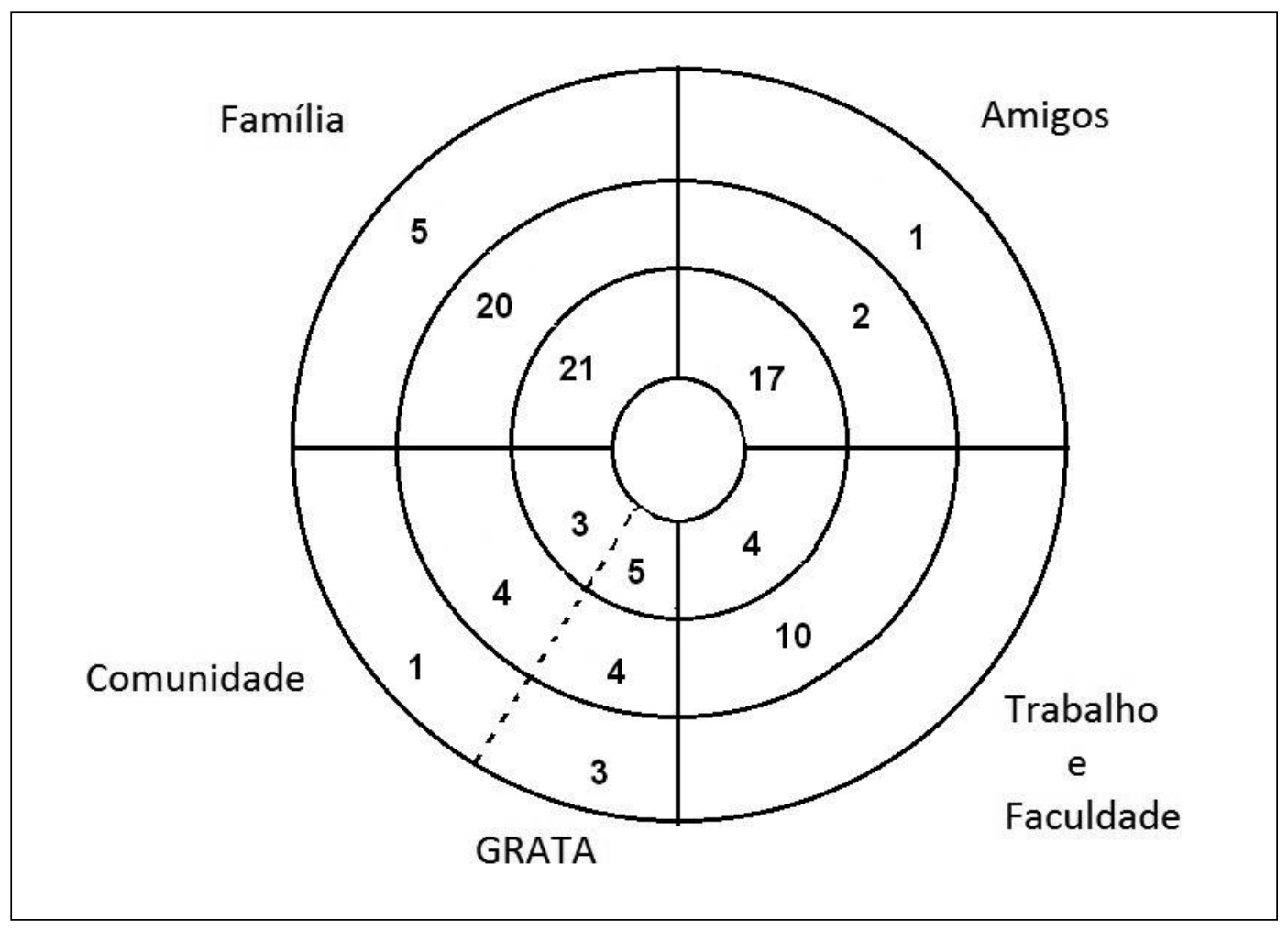

Figura 2. Distribuição dos membros das redes de todas as participantes nos quadrantes dos Mapas de Rede.

Levando-se em conta o objetivo do estudo, foram elaboradas quatro categorias de análise (grafadas em negrito) e suas respectivas subcategorias (grafadas em itálico), cujas nomeações foram definidas a partir da articulação de elementos constituídos pelas regularidades temáticas presentes no depoimento das entrevistadas, dos conceitos teóricos extraídos da literatura especializada e dos termos específicos oriundos dos Mapas de Rede.

\section{C ategoria 1. E strutura e F uncionalidade das Redes Sociais Significativas}

Segundo Orlandi (2011), não há um consenso na literatura acerca do que pode ser considerada uma rede de tamanho grande, médio ou pequeno. Assim, baseando-se no modelo proposto pela referida autora, determinou-se que as redes de tamanho pequeno seriam aquelas nas quais foram apresentados até seis membros, de tamanho médio 
aquelas redes com até 10 membros e, por fim, as redes que englobavam mais de 11 membros foram caracterizadas como grandes. Vale ressaltar que algumas participantes mencionaram "pessoas", "tios", "amigas", sem definir o número de indivíduos, o que impossibilitou a quantificação exata de membros das redes sociais. Nesses casos, foram atribuídos números aproximados de membros por redes.

As redes das participantes do presente estudo apresentaram entre sete (Sissi) e 22 membros (Marina), caracterizando três redes de tamanho médio e três de tamanho grande. No entanto, apesar da quantidade média ou elevada de membros nas redes, nota-se que boa parte deles não exercia papel significativo na vida das participantes. A maioria desempenhava a função de companhia social, que se refere a um tipo de função de ajuda que remete à realização de atividades em conjunto com outras pessoas, podendo consistir simplesmente em estar junto (Sluzki, 1997). Os membros que desempenharam essa função foram: amigas da faculdade e do trabalho, vizinhas, irmãs e irmãos, primos, sobrinhos, tios, cunhado e namorado. As participantes não mantinham um vínculo de apoio significativo com a maioria desses membros, sendo que a frequência dos contatos e o grau de intimidade eram baixos, resultando em relações com pouca intensidade de compromisso.

Em termos de sua composição, as redes apresentavam maior concentração de membros no quadrante da Família, evidenciando predomínio da rede social familiar no contexto de vida das participantes. Os membros da família desempenhavam maior número de funções de ajuda, caracterizando a principal fonte de apoio. A mãe foi o membro que exercia a maior quantidade de funções de ajuda, predominantemente a função de apoio emocional. No entanto, no que diz respeito à multidimensionalidade das funções desempenhadas, evidenciou-se que a mãe também exercia várias outras funções, ainda que de forma esporádica: companhia social, guia cognitivo e de conselhos, serviços e cuidados, ajuda material e financeira, bem como regulação social.

Por fim, as redes sociais representadas graficamente apresentavam elevado grau de densidade e baixo grau de dispersão, uma vez que a maioria dos membros mantinha contato entre si e morava em lugares geograficamente próximos. Considera-se que a articulação entre os membros da rede favorece o acionamento das fontes de apoio social no enfrentamento do quadro da pessoa acometida por uma doença grave (Moré, 2005). No entanto, a análise mais acurada do fornecimento de apoio social e da satisfação das participantes com tal apoio depende de investigação minuciosa dos vínculos estabelecidos, assim como da dinâmica familiar e da rede social de amigos. Os dados relativos a essas outras dimensões das redes serão descritos nas categorias a seguir.

\section{C ategoria 2. Organização da R ede de A poio Familiar}

Ao serem questionadas a respeito das relações familiares, à exceção de Grazieli, as participantes relataram a existência de muitos conflitos, discórdias e brigas. No entanto, apesar da tensão gerada pelas dissensões, as participantes relataram que se sentem apoiadas pela família.

"Mas, assim, apesar de tudo são pessoas, assim que, nunca me abandonaram, sabe? Sempre me apoiaram, sempre estiveram comigo, tudo..." (Tatiana).

"Além do meu pai e da minha mãe, que estiveram o tempo todo me sustentando, me ajudando... [.. .] Sem falar, meu irmão também. Sempre... Nossa! Preocupadíssimo... Sempre tentou me ajudar demais, também"(Paula).

Os relatos foram convergentes no que diz respeito ao fato de que a família tem consciência do TA, porém evita falar abertamente sobre o problema. No que concerne às relações familiares, autores como Bryant-Waugh e Lask (1995), Lane (2002) e McNamara e Loveman (1990), pontuam que as famílias de pacientes com TA geralmente apresentam desarmonia, pouco afeto e respeito entre seus membros, comunicação empobrecida, escassa habilidade para a resolução de conflitos, mães excessivamente controladoras e pais ausentes. Os relatos das participantes e a configuração dos Mapas de Rede corroboram os achados da literatura, uma vez que evidenciaram que as relações familiares eram marcadas por conflitos, brigas e sensação de incompreensão, além de ausência de empatia.

No entanto, apesar dos conflitos relatados pelas participantes, também ficou evidenciado que a família é a principal fonte de apoio percebido por elas, sendo que a mãe foi descrita como figura central no cenário doméstico. Bullock (2004) sugere que, apesar de seus múltiplos formatos e inúmeros significados, a família encontra-se no centro da influência interpessoal. É fonte de trocas mútuas, designa um grupo de referência e um contexto social, assim como uma área de promoção de saúde. Além disso, a autora também pontua que a família, geralmente, é a principal fonte de apoio e cuidado, pois seus componentes estão dispostos a empreender grandes sacrifícios para fornecer cuidados ao membro acometido. Moré (2005) complementa esses achados ao sugerir que a qualidade das relações interpessoais apresenta estreita relação com a história dos vínculos estabelecidos, na intensidade, frequência e mutualidade dos mesmos. Nesse sentido, pode-se considerar que o contexto familiar das participantes, mesmo que caracterizado por relacionamentos disfuncionais, constitui-se como principal contexto de apoio existente em suas vidas, no qual se sentem pertencentes e socializadas.

Em alguns casos (Denise, Juliana e Tatiana), a relação com a mãe era caracterizada pelas participantes como conflitual, marcada por momentos em que a mãe não aceitava o TA e acabava "brigando" ou discutindo com a filha. Esses momentos de brigas e discussões eram vivenciados pelas participantes como incompreensão, ou seja, elas não se sentiam acolhidas e compreendidas pela mãe. No entanto, todas as participantes relataram que sua principal fonte de apoio era a mãe, mesmo aquelas que mantinham relações permeadas por conflitos.

Meus pais, assim... É uma relação boa, sabe? Apesar de meio conflituosa, porque tem muita cobrança, tem 
muita ameaça, tem muita... [. . . A minha mãe, ela é mais assim, explosiva, sabe assim? Ela me cobra muito, ameaça muito assim, sabe? E ela é muito explosiva, assim, sabe... Tudo já... A gente acaba discutindo bastante, tudo... (Tatiana)

Segundo Adami-Lauand (2010), a relação entre mãe e filha com TA de um modo geral se desenvolve a partir de um processo de indiferenciação, de modo que, borrando os contornos que definem as individualidades, mantêm-se vínculos simbióticos. Nesse contexto relacional, resta pouco espaço para diferenciação psíquica e reconhecimento da individualidade. A relação de fusão e indiferenciação comumente é marcada pela ambivalência entre amor e ódio, uma vez que os membros da dupla se amam e desejam a proximidade um do outro, mas, ao mesmo tempo, tentam sem sucesso se separar para adquirirem individuação. Levando-se em conta a dinâmica ambivalente vivenciada pelas mulheres com TA na relação com a figura materna, e considerando também que a mãe é o principal agente de cuidados à saúde (Gutierrez \& Minayo, 2008), é possível compreender como esse modo de vinculação tão conflituoso pode restar, paradoxalmente, como a principal fonte de apoio presente na vida das participantes.

\section{Categoria 3. Rede de A poio de Amigos}

As participantes do presente estudo relataram enfrentar muitas dificuldades no contato interpessoal, e atribuem essa dificuldade ao baixo número de amigos, que constituíram o respectivo quadrante no Mapa de Rede. Trata-se de amigos da infância (Grazieli) ou de longa data (Paula), amigos da faculdade e/ou do trabalho (Sissi, Paula, Juliana e Tatiana) e vizinhas (Denise).

Durante a entrevista, as participantes relataram que não tinham amigos, e sim "colegas", ou seja, pessoas que viam com certa frequência e com quem conversavam superficialmente. Admitiram ter dificuldade em se aproximar das pessoas e estabelecer um vínculo sólido. Essas pessoas denominadas de "colegas" não foram inseridas nos Mapas de Rede por escolha das próprias participantes, que caracterizavam o contato como tão superficial, que tais pessoas não faziam diferença em suas vidas a ponto de poderem ser consideradas parte de suas redes sociais. As únicas exceções a essa tendência foram as duas estudantes universitárias, que inseriram no círculo de "Relações Sociais" também algumas amigas da faculdade, embora tenham frisado que se tratam de amigas com quem elas não têm um vínculo afetivo propriamente, mas com quem mantêm contato diário.

"Não, tenho alguns, assim, mas que eu não digo, assim, que são amigos... Colegas eu acho que você tem muitos, mas amigos, amigos, dizer que eu posso contar sempre com eles, eu acho que são muito poucos" (Sissi).

Como consequência da ausência de atividades exercidas fora do ambiente de trabalho e estudo, foram inseridos nos Mapas de Rede apenas os indivíduos que faziam parte dessas atividades, além da família e de pessoas da igreja (Grazieli e Paula). A escassez de relações interpessoais, evidenciada por meio da análise dos Mapas de Rede, pode ser compreendida como ilustração gráfica da dificuldade que as participantes apresentam em manter contato com as pessoas do meio em que vivem, restringindo assim o tamanho e a densidade de suas redes sociais (Sluzki, 1997). Em consequência dessa restrição, a função da rede e o apoio social disponível para as participantes ficam comprometidos, uma vez que esses recursos estão relacionados com a qualidade das relações interpessoais (Moré, 2005).

Resende et al. (2006) sugerem que o apoio social pode ser caracterizado como a ajuda material, emocional ou de informação para enfrentar situações que envolvem tensão emocional, sendo que essa ajuda é proveniente da rede social de apoio que o indivíduo possui. Essa ajuda também está relacionada com a possibilidade de manutenção da saúde e bem-estar do indivíduo, uma vez que as relações sociais contribuem para dar sentido à vida de seus membros (Sluzki, 1997). Ou seja, o olhar e as ações dos outros, direcionados ao indivíduo, favorecem a organização da identidade, e disso deriva a experiência de "existir para alguém" ou de "servir para alguma coisa" que, por sua vez, dá sentido e estimula a manutenção das práticas de cuidados à saúde, visando à continuidade da própria vida (Sluzki, 1997).

As participantes deste estudo manifestaram seu sentimento de irrelevância, isto é, de serem insignificantes para o outro, de permanecerem invisíveis e de não serem reconhecidas em suas qualidades. Juliana associou claramente esse sentimento persistente de "inexistência" pessoal com o início de seus sintomas anoréxicos: "É contraditório, mas eu queria desaparecer para poder aparecer". Por outro lado, são muito sensíveis ao serem ignoradas. O déficit de autoestima pode gerar dificuldade de contato com os demais, o que aprofunda a vulnerabilidade e uma percepção diminuída de suas capacidades, conduzindo à vivência distorcida de desaparição do self. Esses achados corroboram o estudo de Shea e Pritchard (2007), que mostrou que a autoestima rebaixada é um dos principais preditores dos TAs. Sluzki (1997) também sugere que "a rede proporciona uma retroalimentação cotidiana a respeito dos desvios de saúde que favorece os comportamentos corretivos" (p. 76), ou seja, as relações sociais servem para monitorar a saúde e as consultas a especialistas, assim como as atividades pessoais que estão relacionadas, de forma positiva, com a sobrevivência do indivíduo, tais como: rotina dietética, exercícios físicos, sono, adesão ao tratamento medicamentoso, entre outras práticas de autocuidado.

Levando-se em conta esses pressupostos, é possível postular que a dificuldade que as participantes do presente estudo manifestam no contato interpessoal, assim como a escassez e falta de densidade da rede social demonstradas por meio dos Mapas de Rede, podem estar relacionadas com a manutenção e/ou intensificação do quadro psicopatológico. Ou seja, uma vez que o número das relações sociais é reduzido e sua qualidade empobrecida, as participantes não têm a possibilidade de dar sentido à sua própria saúde, pois há poucas pessoas em suas redes que 
podem estimulá-las a manter os autocuidados, monitorando a adesão ao tratamento e a hábitos de vida saudáveis. Isso acentua a dificuldade que elas sentem em manter atividades cotidianas de autocuidado, especialmente de seguir o plano alimentar sem apoio externo reiterado no cotidiano.

\section{Categoria 4. Dificuldades nos R elacionamentos A fetivos e Conjugais}

Apenas duas participantes relataram que estavam namorando. Referiram que se sentiam muito satisfeitas com o relacionamento afetivo (Grazieli e Tatiana) e incluíram os namorados nos seus respectivos Mapas de Rede. Outras duas (Denise e Paula) já foram casadas, e relataram que o relacionamento era bastante conturbado, marcado por infidelidades conjugal e brigas constantes. Denise tem dois filhos e havia se separado do marido dois meses antes da realização da entrevista, porém não estava divorciada judicialmente, mantendo contato muito próximo com o ex-cônjuge. No entanto, relatou já estar interessada em outro homem e não incluiu o ex-marido no Mapa de Rede. Paula casou-se aos 20 anos de idade, e permaneceu casada durante quatro anos. Não teve filhos.

Sissi relatou que já manteve um relacionamento afetivo prolongado, porém muito conflituoso. Atribui esses conflitos ao fato de ela própria ser "muito difícil de lidar", por ser "muito controladora", ou seja, os aspectos típicos de seu funcionamento emocional dificultavam o desenvolvimento de um relacionamento harmonioso. Por fim, Juliana relatou nunca ter namorado "sério", mas manifestou o desejo de que isso acontecesse logo.

"Ai, eu sempre fui muito controladora. Então, sempre era assim, eu controlando" (risos). (Sissi).

As dificuldades relatadas pelas participantes, no plano de seus relacionamentos conjugais e afetivos, parecem ser reflexo do efeito produzido por seu funcionamento emocional sobre os parceiros, e acabam incrementando as barreiras encontradas para constituírem e manterem vínculos amorosos. A desorganização pessoal parece encontrar expressão nos relacionamentos disfuncionais, que acabam fomentando discórdias e promovendo rupturas nas relações interpessoais.

Sluzki (1997) sugere que o casamento é um ritual que formaliza a consolidação de novas redes sociais dos membros do casal, já que costuma haver junção de elementos das redes anteriores de ambos. Quando acontece a separação, além da perda vincular central (perda do cônjuge), dá-se a dissolução de vários outros vínculos e projetos, inclusive de pessoas que até então eram figuras significativas na vida dos cônjuges. Além disso, novos vínculos são agregados à rede de cada indivíduo, pessoas que não conheceram o casal anterior e que, portanto, não incluem na sua percepção do sujeito a história de seu casamento, e nas quais o sujeito vê apenas a sua nova identidade de "separado, divorciado ou novamente solteiro" (Sluzki, 1997, p. 106).

Assim, a separação, o divórcio ou o término de um namoro exercem influência nas redes sociais significa- tivas dos indivíduos, alterando suas fontes de apoio: as pessoas que antes costumavam ser significativas na vida do sujeito passam a se afastar em função da separação do casal, abrindo espaço para que novos membros passem a fazer parte da rede pessoal. A perda desses vínculos, como pontuou Sluzki (1997), leva à reconfiguração das redes, ou seja, obriga o sujeito a realizar diversas mudanças em sua rotina e a dar conta de obrigações que antes podiam ser divididas com o cônjuge (por exemplo, a educação dos filhos).

... Então, depois que aconteceu isso foi mais um motivo para eu pedir a separação. Só que eu tinha medo de me separar e não ter condições de criar a minha filha sozinha, e depois eu vi que não, que eu sou capaz de criar, que eu tinha capacidade de criar meus filhos sozinha. (Denise)

No caso das mulheres com TAs, pode-se pensar que as redes significativas - que já eram escassas - tornaram-se mais restritas após os remanejamentos, uma vez que as participantes deste estudo mostram dificuldade na formação de vínculos e, consequentemente, na abertura de espaço para que novas pessoas adentrem suas redes. Essa restrição do número de figuras significativas na vida das participantes pode levar à diminuição do potencial de apoio recebido e, juntamente com as vivências de afetos negativos decorrentes da perda do vínculo conjugal, podem contribuir para o agravamento dos sintomas do TA, uma vez que as redes e o apoio social exercem influência nas emoções do indivíduo - que, por sua vez, afetam a saúde mental e física do mesmo (Bullock, 2004).

\section{Considerações Finais}

O presente estudo teve como objetivo investigar o modo como se configuram as redes sociais de mulheres com TAs, assim como o apoio social percebido por elas e as possíveis influências dessas relações sobre a evolução do quadro psicopatológico. Considerando esses objetivos, constatou-se que as redes sociais das participantes apresentavam tamanhos médios ou grandes, mas eram pouco diversificadas, com predominância dos membros da família. As outras pessoas incluídas nas redes significativas eram as que as participantes conheciam, mas com quem mantinham apenas contato social ou distante, e que não desempenhavam funções significativas de ajuda no enfrentamento do TA. No círculo constituído pelas "Relações Íntimas", por sua vez, foram incluídos alguns membros da família e poucos amigos íntimos, que foram nomeados e descritos como significativas fontes de apoio social.

Evidenciou-se que o apoio social principal era proveniente das relações familiares, ainda que estas fossem marcadas por conflitos, tensões e discussões frequentes. A predominância da rede familiar, com quem as participantes mantinham vínculos naturalizados por laços de sangue antes da instalação do TA, juntamente com a escassez de amigos, colegas de trabalho e indivíduos de outras redes sociais, indicam a existência de dificuldades no estabe- 
lecimento de vínculos consistentes e na manutenção de relacionamentos afetivos e conjugais duradouros, o que parece comprometer a qualidade dessas relações e desencorajar a aproximação a pessoas do meio.

Nesse sentido, considera-se necessária a problematização da qualidade dos vínculos significativos que as mulheres com esses quadros psicopatológicos graves e persistentes estabelecem com os membros de suas redes sociais. Vale ressaltar a importância dos profissionais da área incluírem os membros dessas redes no contexto da assistência, buscando melhorar as relações e explorar o potencial de apoio das mesmas. Tal aprimoramento na assistência pode resultar em melhor qualidade de vida das pacientes, uma vez que esta se encontra intimamente relacionada com a qualidade das relações sociais do indivíduo.

Uma vez que o cuidado integral das pacientes com TAs não se restringe apenas ao acompanhamento nutricional, psiquiátrico e psicológico de forma isolada, acredita-se que os elementos resultantes do presente estudo apontam para a necessidade de que a equipe multidisciplinar dimensione os impactos que as redes sociais acarretam na saúde dos indivíduos. Esse conhecimento pode oferecer subsídios valiosos para aprimorar os recursos de tratamento dos TAs, orientando, especialmente, os modos de incluir as redes de apoio na assistência, de modo a prevenir novos agravos à saúde e promover o bem-estar.

Outro aspecto a ser destacado a respeito da relevância do trabalho com redes diz respeito à distribuição das responsabilidades do cuidado. Ao considerar as reais possibilidades de cada um, o profissional pode valorizar as relações que propiciam melhoria na qualidade de vida do indivíduo e, assim, conseguir angariar aliados na família do paciente, ou na própria comunidade, que devem ser valorizados e incluídos como unidade de tratamento.

Cabe salientar também o valor do uso do Mapa de Rede como um instrumento relevante de pesquisa e intervenção na área da saúde, pois possibilita a verificação do tamanho e da qualidade das redes de apoio social. O processo de construção do Mapa permite que o indivíduo visualize o tipo de relações que ele vem estabelecendo ao longo de sua vida, o que estimula a reflexão e a auto-observação. Isso pode ter um efeito terapêutico. Levando-se em conta os resultados obtidos com a utilização do Mapa de Rede no presente estudo, conjugado com a entrevista semiestruturada, é possível considerá-lo como um instrumento que se mostrou particularmente útil na identificação da necessidade de apoio e na visualização dos recursos disponíveis nas redes de indivíduos que se encontram em situação de doença crônica, o que possibilita o planejamento de intervenções que incluam a família e outras pessoas que constituem a rede significativa da pessoa acometida.

No entanto, vale ressaltar que as relações humanas, assim como a estrutura das redes sociais significativas e a percepção do apoio social recebido, apresentam uma dinamicidade intrínseca, ou seja, encontram-se em constante movimento de renovação e transformação. Pessoas são incluídas nas redes sociais como fontes importantes de apoio e, algum tempo depois, devido a rompimentos nas relações ou às possíveis adversidades da vida, acabam sendo afastadas daquela rede, modificando sua estrutura e abrindo espaço para que outras pessoas possam fazer parte dela. Nesse sentido, é válido ressaltar que, apesar do presente estudo apresentar dados expressivos acerca das redes sociais, dos vínculos estabelecidos com pessoas significativas e da dinâmica das relações familiares de mulheres com TAs, os resultados encontrados são pontuais e não podem ser considerados estáticos, mas sim como concernentes a um momento específico da trajetória de vida das participantes. Além disso, a dificuldade de quantificação dos membros das redes também se apresentou como uma limitação do presente estudo, uma vez que implicou em trabalhar com números aproximados, e não exatos, das pessoas que constituíam essas redes.

\section{R eferências}

Abreu, N., \& Cangelli, R. C., Filho. (2004). Anorexia nervosa e bulimia nervosa: Abordagem cognitivo-construtivista de psicoterapia. Revista de Psiquiatria Clínica, 31(4), 177-183.

Adami-Lauand, C. B. (2010). As experiências alimentares de mães com filhas portadoras de transtornos alimentares: Investigando a transgeracionalidade (Dissertação de mestrado, Universidade de São Paulo, Escola de Enfermagem de Ribeirão Preto, SP, Brasil).

Andrade, G. R. B., \& Vaitsman, J. (2002). Apoio social e redes: Conectando solidariedade e saúde. Ciência \& Saúde Coletiva, 7(4), 925-934.

Andrade, T. F., \& Santos, M. A. (2009). A experiência corporal de um adolescente com transtorno alimentar. Revista Latinoamericana de Psicopatologia Fundamental, 12(3), 454-468.

Associação Americana de Psiquiatria. (2003). DSM-IV-TR ${ }^{T M}$ - Manual diagnóstico e estatístico de transtornos mentais: Texto revisado (4. ed. rev., C. O. Dornelles, Trad.). Porto Alegre, RS: Artmed.

Bogdan, R. C., \& Biklen, S. K. (1994). Investigação qualitativa em educação: Uma introdução à teoria e aos métodos (M. J. Alvarez, S. B. Santos, \& T. M. Batista, Trads.). Porto, Portugal: Ciência da Educação.

Borges, N. J. B. G., Sicchieri, J. M. F., Ribeiro, R. P. P., Marchini, J. S., \& Santos, J. E. (2006). Transtornos alimentares: Quadro clínico. Medicina (Ribeirão Preto), 39(3), 340-348.

Bryant-Waugh, R., \& Lask, B. (1995). Eating disorders: An overview. Journal of Family Therapy, 17, 13-30.

Bullock, K. (2004). Family social support. In P. J. Bomar (Ed.), Promoting health in families: Apllying reserach and theory to nursing practice (pp. 141-161). Philadelphia, PA: Saunders.

Cassin, S. E., \& Von Ranson, K. M. (2005). Personality and eating disorders: A decade in review. Clinical Psychology Review, 25(7), 895-916.

Castro-Fornieles, J., Gual, P., Lahortiga, F., Gila, A., Casulà, V., Fuhrmann, C., ... Toro, J. (2007). Self-oriented perfectionism in eating disorders. International Journal of Eating Disorders, 40(5), 562-568.

Claudino, A. M., \& Borges, M. B. F. (2002). Critérios diagnósticos para os transtornos alimentares: Conceitos em evolução. Revista Brasileira de Psiquiatria, 24(3), 7-12. 
Custódio, Z. A. O. (2010). Redes sociais no contexto da prematuridade: Fatores de risco e de proteção para o desenvolvimento da criança ao longo dos seus dois primeiros anos de vida (Tese de doutorado, Programa de Pós-Graduação em Psicologia, Universidade Federal de Santa Catarina, Florianópolis, SC, Brasil).

Guest, G., Bunce, A., \& Johnson, L. (2006). How many interviews are enough?: An experiment with data saturation and variability. Field Methods, 18(1), 59-82.

Gutierrez, D. M. D., \& Minayo, M. C. S. (2008). Família, redes sociais e saúde: O imbricamento necessário. In Anais do VIII Fazendo Gênero: Corpo, violência e poder. Florianópolis, SC: Universidade de Santa Catarina.

Kaye, W. (2008). Neurobiology of anorexia and bulimia nervosa. Physiology \& Behavior, 94(1), 121-135.

Lane, R. C. (2002). Anorexia, masochism, self-mutilation, and autoerotism: The spider mother. Psychoanalitic Review, 89(1), 101-123.

Leonidas, C., \& Santos, M. A. (2012). Imagem corporal e hábitos alimentares na Anorexia Nervosa: Uma revisão integrativa da literatura. Psicologia: Reflexão e Crítica, 25(3), 550-558.

Marcos, Y. Q., \& Cantero, M. C. T. (2009). Assesment of social support dimensions in patients with eating disorders. The Spanish Journal of Psychology, 12(1), 226-235.

McNamara, K., \& Loveman, C. (1990). Differences in family functioning among bulimics, repest dieters, and nondieters. Journal of Clinical Psychology, 46(3), 518-523.

Minayo, M. C. S. (1994). O desafio do conhecimento: Pesquisa qualitativa em saúde (3. ed.). São Paulo, SP: Hucitec.

Moré, C. L. O. O. (2005). As redes pessoais significativas como instrumento de intervenção psicológica no contexto comunitário. Paidéia (Ribeirão Preto), 15(31), 287-297.

Nilsson, K., Sundbom, E., \& Häglöf, B. (2008). A longitudinal study of perfectionism in adolescent onset anorexia nervosa - Restricting type. Europen Eating Disorders Review, 16(5), 386-394.

Oliveira, E. A., \& Santos, M. A. (2006). Perfil psicológico de pacientes com anorexia e bulimia nervosas: Ótica do psicodiagnóstico. Medicina (Ribeirão Preto), 39(3), 353-360.

Orlandi, R. (2011). Participação da rede social significativa de mulheres que vivem e convivem com o HIV no enfrentamento da soropositividade (Tese de doutorado, Programa de Pós-Graduação em Psicologia, Universidade Federal de Santa Catarina, Florianópolis, SC, Brasil).

Paccola, A. T. F. (2006). Escuta do psiquiatra: Sinais e sintomas de anorexia nervosa e bulimia nervosa. Medicina (Ribeirão Preto), 39(3), 349-352.

Peres, R. S., \& Santos, M. A. (2006). Contribuições do Desenho da Figura Humana para a avaliação da imagem corporal na anorexia nervosa. Medicina (Ribeirão Preto), 39(3), 361-370.

Resende, M. C., Bones, V. M, Souza, I. S., \& Guimarães, N. K. (2006). Rede de relações sociais e satisfação com a vida de adultos e idosos. Psicologia para a América Latina, 5. Recuperado em http://pepsic.homolog.bvsalud.org/scielo. php?script=sci_arttext\&pid=S1870-350X2006000100015\& $\operatorname{lng}=\mathrm{pt} \& \mathrm{nrm}=\mathrm{iso}$

Rosa, B. P., \& Santos, M. A. (2011). Comorbidade entre bulimia e transtorno de personalidade borderline: Implicações para o tratamento. Revista Latinoamericana de Psicopatologia Fundamental, 14(2), 268-282.

Santos, A. C. W. (2009). Mulheres, violência, rede de serviços de referência e suporte psicosocial (Dissertação de mestrado, Programa de Pós-Graduação em Psicologia, Universidade Federal de Santa Catarina, Florianópolis, SC, Brasil).
Scorsolini-Comin, F., Souza, L. V., \& Santos, M. A. (2010). A construção de si em um grupo de apoio para pessoas com transtornos alimentares. Estudos em Psicologia (Campinas), 27(4), 467-478.

Shea, M. E., \& Pritchard, M. E. (2007). Is self-esteem the primary predictor of disordered eating? Personality and Individual Differences, 42(8), 1527-1537.

Silva, I., Pais-Ribeiro, J., Cardoso, H., Ramos, H., Carvalhosa, S. F., Dias, S., \& Gonçalvez, A. (2003). Efeitos do apoio social na qualidade de vida, controle metabólico e desenvolvimento de complicações crônicas em indivíduos com diabetes. Psicologia, Saúde e Doenças, 4(1), 21-32.

Sluzki, C. E. (1997). A rede social na prática sistêmica: Alternativas terapêuticas (C. Berliner, Trad.). São Paulo, SP: Casa do Psicólogo.

Souza, L. V., \& Santos, M. A. (2007). Anorexia e bulimia: Conversando com as famílias. São Paulo, SP: Vetor.

Souza, L. V., \& Santos, M. A. (2009). A construção social de um grupo multifamiliar no tratamento dos transtornos alimentares. Psicologia: Reflexão e Crítica, 22(3), 317-26.

Souza, L. V., \& Santos, M. A. (2010). A participação da família no tratamento dos transtornos alimentares. Psicologia em Estudo (Maringá), 15(2), 285-294.

Vieira, N. G. V., Filho, \& Nóbrega, S. M. (2004). A atenção psicossocial em saúde mental: Contribuição teórica para o trabalho terapêutico em rede social. Estudos de Psicologia (Natal), 9(2), 373-379.
Recebido: 18/07/2011

$1^{a}$ revisão: 30/03/2012

$2^{a}$ revisão: $22 / 05 / 2012$

$3^{a}$ revisão: 26/06/2012

Aceite final: 26/06/2012 